\title{
Estudo de Adaptação e Características Psicométricas da Versão Portuguesa da Adolescent Coping Scale - Escala de Coping para Adolescentes
}

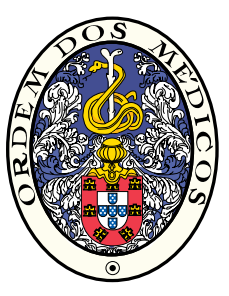

\author{
Adaptation and Psychometric Proprieties Study for the Portuguese Version of \\ the Adolescent Coping Scale - Escala de Coping para Adolescentes
}

\author{
Diogo Frasquilho GUERREIRO ${ }^{1}$, Diana CRUZ², Maria Luísa FIGUEIRA ${ }^{1}$, Daniel SAMPAIO ${ }^{1}$ \\ Acta Med Port 2014 Mar-Apr;27(2):166-180
}

\section{RESUMO}

Introdução: O coping é um processo psicológico que leva ao ajustamento individual perante situações de stress. A Adolescent Coping Scale é um instrumento de investigação e uma ferramenta clínica, amplamente utilizada. O presente estudo tem como objectivos desenvolver uma versão Portuguesa da Adolescent Coping Scale e analisar as estratégias e estilos de coping dos jovens da nossa amostra. Material e Métodos: Um questionário anónimo compreendendo a Adolescent Coping Scale obteve respostas de 1713 alunos (56\% do sexo feminino, com idades compreendidas entre os 12 e os 20 anos e uma média etária de 16). O estudo de validade da escala contemplou: análise em componentes principais e avaliação da consistência interna; análise confirmatória através de modelo de equações estruturais. Posteriormente, foram comparadas por género as estratégias e estilos de coping da amostra (testes $t$ para amostras independentes).

Resultados: A estrutura final da adaptação da Adolescent Coping Scale reteve 70 itens, que avaliam 16 estratégias de coping agrupadas em três estilos distintos. As escalas apresentaram bons valores de consistência interna (alfas de Cronbach compreendidos entre 0,63 e 0,86, com a exceção de uma dimensão que apresentou um valor de 0,55 ) e o modelo confirmatório demonstrou bom fit (goodness of fit index compreendidos entre 0,94 e 0,96). Foram eliminadas duas estratégias de coping por motivos estatísticos (ausência de saturação de itens suficientes nas dimensões correspondentes). Verificámos que o estilo de coping focado na resolução do problema é aquele maioritariamente utilizado pelos adolescentes da nossa amostra, em ambos os sexos. No sexo feminino observaram-se valores médios mais elevados nos estilos de coping não produtivo e de referência a outros.

Discussão: A versão adaptada apresenta elevada semelhança com a escala original, com alterações minor espectáveis tendo em conta que o coping é influenciado por variáveis culturais, geográficas e sócioeconómicas.

Conclusão: O presente estudo representa uma parte importante do protocolo de validação Portuguesa da Adolescent Coping Scale, nomeadamente a sua adaptação linguística, estudo da consistência interna e da estrutura fatorial.

Palavras-chave: Adaptação Psicológica; Adolescente; Questionários; Psicometria; Portugal.

\section{ABSTRACT}

Introduction: Coping is a psychological process that prompts the individual to adapt to stressful situations. The Adolescent Coping Scale is a widely used research and clinical tool. This study aimed to develop a Portuguese version of the Adolescent Coping Scale and to analyze the strategies and coping styles of young people in our sample.

Material and Methods: An anonymous questionnaire comprising the Adolescent Coping Scale was submitted and replied by 1713 students (56\% female, from 12 to 20 years, average age 16$)$ The validity study of the scale included: principal component and reliability analysis; confirmatory analysis using structural equation modelling Subsequently, a gender comparison of both the strategies and the coping styles was conducted through independent samples t tests.

Results: The final structure of the Adolescent Coping Scale adaptation retained 70 items assessing 16 coping strategies grouped into three major styles. The scales showed good internal consistency (Cronbach alpha values between 0.63. and 0.86, with the exception of one dimension that as shown a value of 0.55 ) and the confirmatory model showed a good fit (goodness of fit index values between 0.94 e 0.96). Two coping strategies were eliminated on statistical grounds (insufficient saturations of items in the corresponding dimensions). We found that the style of coping focused on problem solving is the most used by youths from our sample, in both sexes. Females had higher mean values in non-productive coping style and reference to others.

Discussion: This adapted version has high similarity with the original scale, with expectable minor changes, given that coping is influenced by cultural, geographical and socio-economic variables.

Conclusion: The present study represents an important part of the validation protocol Portuguese Adolescent Coping Scale, including its linguistic adaptation and its internal consistency and factor structure studies.

Keywords: Adaptation, Psychological; Adolescent; Questionnaires; Portugal; Psychometrics.

\section{INTRODUÇÃO}

O coping é definido por Lazarus e Folkman ${ }^{1}$ como 'esforços comportamentais e cognitivos, em mudança constante, que visam gerir exigências internas ou externas específicas, consideradas como excedendo os recursos pessoais'. É assim um factor estabilizador, ou seja, que facilita o ajustamento individual ou a adaptação, quando se está perante eventos stressantes. ${ }^{2}$ Como evidência da adaptação encontramos o bem-estar, o funcionamento social e a saúde somática. A eficácia do coping é transacional, ou seja, depende da interação entre várias variáveis, como as

1. Departamento de Psiquiatria. Faculdade de Medicina. Universidade de Lisboa. Lisboa. Portugal.

2. Faculdade de Psicologia. Universidade de Lisboa. Lisboa. Portugal.

Recebido: 29 de Julho de 2013 - Aceite: 20 de Novembro de 2013 | Copyright @ Ordem dos Médicos 2014 
características da situação e os recursos do indivíduo, o objectivo do processo de coping em si mesmo e determinantes do indivíduo. ${ }^{1}$ As estratégias de coping são antecipadas por mecanismos de avaliação pessoal e ambiental. A pessoa ativa processos cognitivos para identificar a situação crítica com a qual se depara, verifica as suas condições atuais, experiências eficazes e ineficazes anteriores, nível e grau de risco envolvido nesta e analisa os recursos disponíveis e as alternativas possíveis para lidar com o problema. ${ }^{3} \mathrm{O}$ processo de coping pode variar quando o problema é percebido em casa ou na escola, por exemplo, sendo que nestes contextos existe uma grande influência do tipo de relações interpessoais (nas quais coexistem questões de hierarquia e poder). Os adolescentes utilizam estratégias diferentes para enfrentar problemas com seu grupo de pares ou problemas com adultos (por exemplo: pais ou professores). Até à data a maioria das investigações sobre coping na adolescência têm sido fundamentadas por este modelo teórico, ${ }^{4}$ geralmente conhecido como 'modelo transacional de coping'. ${ }^{3}$

Os agrupamentos preferenciais de estratégias de coping que os indivíduos utilizam face a stressores podem definir dois grandes estilos de coping ${ }^{3,4}$ : o coping focado na emoção (incluindo estratégias como distanciamento e evitamento) que tem como objectivo a regulação emocional perante uma situação stressora; e o coping focado na resolução do problema que tem como objectivo lidar com o problema que está a causar a situação de desregulação. Outros investigadores defendem que as estratégias de coping são melhor agrupadas em três estilos ${ }^{4}$ : focado na resolução do problema que envolve tentativas para lidar diretamente com o stressor; referência a outros envolvendo a consulta e pedido de ajuda a pessoas significativas da sua rede de apoio social e; não produtivo que se baseia na utilização de estratégias de evitamento, isto é, que não confrontam diretamente o problema.

A adolescência é uma etapa do ciclo de vida inerentemente associada a vários stressores,${ }^{5}$ o que implica inúmeros ajustamentos (como por exemplo: face às mudanças fisiológicas; à evolução das capacidades cognitivas; à experimentação social; etc.). ${ }^{6,7}$ Trata-se também de uma altura em que existe uma oportunidade única para compreender e estimular capacidades de coping, o que dá relevo à importância da avaliação das estratégias e estilos de coping utilizados pelos jovens.

No entanto, o modo como cada indivíduo recorre às suas estratégias de coping varia consoante o género: as raparigas têm tendência para usar estratégias mais passivas centradas na regulação emocional ou na procura de apoio social, enquanto os rapazes usam mais estratégias ativas, lúdicas ou evitantes. ${ }^{8,9}$ Adicionalmente, o uso de certas estratégias é sujeito a variações conforme as culturas, as zonas geográficas ou os estratos socioeconómicos (por exemplo, num estudo comparativo de adolescentes Alemães, Australianos, Colombianos e Palestinianos observou-se que 'fazer atividade física' é a estratégia mais utilizada pelos jovens Alemães, enquanto que no caso dos
Palestinianos esta está em $16^{\circ}$ lugar no ranking das estratégias utilizadas). ${ }^{10-12}$

De um ponto de vista médico, os processos de coping são também de elevada relevância, não só nas doenças mentais ${ }^{13}$ mas também numa variedade de doenças físicas, em que cada vez mais importância se tem dado a este tema. ${ }^{14,15}$ A título de exemplo, doenças como a diabetes tipo 1 , a dor crónica e as neoplasias na adolescência, têm sido foco de investigação nesta área, demonstrando-se o papel do coping no ajustamento e no prognóstico das mesmas. ${ }^{15}$

A avaliação do coping iniciou-se com uma orientação centrada no adulto ${ }^{16,17}$ e apenas há cerca de 30 anos se desenvolveu um interesse crescente pelo estudo do coping na adolescência, levando ao desenvolvimento de vários instrumentos para a medição do coping nesta faixa etária. ${ }^{4}$ Numa importante revisão de literatura sobre esta temática, Compas et $a^{18}$ fazem uma síntese de todas as escalas criadas, desde 1988, para avaliar o coping, num total de 20 instrumentos, que têm em comum o facto de pedirem ao participante para indicar como lida, ou lidaria, com uma situação de stress, hipotética ou específica. Na maioria das medidas, os procedimentos estatísticos têm sido descurados, sobretudo devido à posição crítica de que '...procedimentos psicométricos como consistência interna e técnicas de análise factorial poderão ter utilidade limitada na avaliação da adequação da medição do coping.' ${ }^{19}$

A Adolescent Coping Scale (ACS) é tanto um instrumento de investigação como de utilidade clínica ${ }^{20}$ que pode ser administrada por técnicos de saúde e de educação. Desenvolvida por Frydenberg e Lewis com adolescentes Australianos, trata-se de um instrumento muito relevante na medição de variáveis de coping, de forte validade psicométrica ${ }^{16,20}$ e extensamente utilizado em vários estudos a nível internacional. ${ }^{4} \mathrm{~A}$ adaptação portuguesa desta escala poderá ser importante na prática clínica e na investigação, uma vez que não parece haver nenhuma adaptação portuguesa publicada até à data.

O objectivo do presente estudo é proceder à adaptação Portuguesa da ACS - Escala de Coping para Adolescentes (versão longa), já adaptada e validada em diversas culturas e línguas. ${ }^{21-25}$ Os objetivos específicos do estudo são (1) analisar a estrutura factorial da ACS numa amostra de adolescentes Portugueses, considerando a provável existência de especificidades culturais e linguísticas; (2) estudar a sua consistência interna e realizar a análise factorial confirmatória; (3) descrever e analisar as diferenças de sexo quanto às estratégias e estilos de coping da ACS, na versão Portuguesa.

\section{MATERIAL E MÉTODOS}

A população alvo foi definida como alunos de escolas públicas da zona metropolitana de Lisboa, com idades compreendidas entre os 12 e os 20 anos (que frequentavam presentemente o $8^{\circ}, 10^{\circ}$ ou $12^{\circ}$ ano). Todas as escolas que incluíam turmas destes anos lectivos da área metropolitana de Lisboa (de acordo com dados fornecidos pelo Ministério da Educação) foram convidadas para participar 
neste estudo. De um total possível de 57, 14 concordaram em participar, correspondendo a uma taxa de recrutamento das escolas de $24 \%$, taxa semelhante ao encontrado noutros estudos deste género. ${ }^{26,27} \mathrm{O}$ tamanho da amostra foi calculado com base no objectivo principal do estudo, tendo sido considerado que uma amostra acima de 1500 seria apropriada, tendo poder estatístico suficiente para os estudos de adaptação da ACS. Para esse efeito foram entregues 2100 questionários.

\section{Medidas}

Variáveis sócio-demográficas - Os dados sócio-demográficos foram acedidos através de um questionário criado no âmbito de um estudo mais abrangente ${ }^{28}$ e que incluiu questões acerca do sexo, idade, estrutura familiar e desempenho escolar.

Coping - A Adolescent Coping Scale (ACS), ${ }^{20}$ versão longa e geral, foi utilizada para avaliar a forma como os jovens 'reagem habitualmente em situação de dificuldade'. Este questionário de auto-relato demora 10/15 minutos a completar e é constituído por 79 questões fechadas (e uma aberta a estratégias não abordadas) que avaliam 18 estratégias de coping conceptualmente e empiricamente distintas, agrupadas em três estilos de coping: focado na resolução do problema; referência a outros e; coping não produtivo. ${ }^{16,20}$ Os 79 itens são avaliados através de uma escala de Likert de cinco pontos (de 1 = Não se aplica ou não faço; a 5 = Faço muito frequentemente). $\mathrm{O}$ instrumento tem duas formas: a específica, em que o participante ou o investigador nomeia determinada situação e todo o questionário é preenchido com referência a essa situação; e a geral, em que o participante baseia as suas respostas na forma como considera reagir habitualmente perante problemas ou dificuldades. A forma específica pode ser utilizada para minimizar determinantes situacionais, enquanto na forma geral se observa como determinado indivíduo utiliza os processos de coping para lidar com um vasto número de situações. ${ }^{4}$

\section{Procedimento}

Tradução e contra-tradução da ACS - Uma vez obtidas as autorizações (dos autores e da editora proprietária da ACS) para proceder à adaptação Portuguesa da escala, este processo decorreu em várias etapas. A primeira fase consistiu na tradução e contra-tradução da ACS por um psiquiatra e uma professora de línguas germânicas, inglesa e bilingue, em condições de dupla ocultação. A contra-tradução foi submetida à avaliação dos autores originais e à avaliação por peritos na área.

Finalmente, procedemos também a um pré-teste do questionário final (após aprovação do Ministério da Educação e após consentimento informado) em 22 alunos do $9^{\circ}$ e $11^{\circ}$ ano de escolaridade. Seguiu-se um focus group com a participação dos mesmos jovens, no sentido de averiguar a clareza da instrução, o tempo médio de preenchimento, a clareza semântica e a compreensão unívoca dos itens, e o próprio formato de resposta. Desta análise, emergiram especificidades culturais decorrendo delas a necessidade de realizar adaptações na linguagem.

Procedimentos de administração - Obtidas as autorizações do Conselho de Ética da Faculdade de Medicina da Universidade Lisboa e do Ministério da Educação, foram expostos os objectivos do estudo, por escrito, aos diretores escolares e aos encarregados de educação, solicitando-se o seu consentimento informado para a participação dos educandos.

Foram entregues 2100 questionários nas escolas participantes. No dia do preenchimento dos mesmos, os alunos estavam acompanhados de um professor (com quem os investigadores reuniram previamente) que orientou o processo e zelou pelo anonimato dos respondentes. Posteriormente, todos os questionários foram selados pelo professor e recolhidos pelos investigadores.

Procedimento estatístico - Inicialmente, procedeu-se a uma análise factorial exploratória - Análise em Componentes Principais (ACP) - através do Software SPSS 19. A opção por uma análise exploratória decorre de: 1) inexistência, do nosso conhecimento, de estudos Portugueses desta medida; 2) potenciais diferenças associadas a diferentes culturas (ex. Australiana vs. Portuguesa) e diferenças socioeconómicas verificadas nos últimos 20 anos.

A estratégia de análise de dados foi a descrita pelos autores originais no seu estudo factorial e consiste em efetuar a ACP em três etapas que correspondem a três partes da escala, constituídas aleatoriamente.

A segunda etapa metodológica consistiu em verificar - através de nova ACP - se as dimensões de coping encontradas se organizavam em estilos gerais de coping, tal como na estrutura original. ${ }^{20}$

Em ambas as etapas foram calculados os valores de alfa de Cronbach (coeficientes de fiabilidade), sendo considerados valores acima de 0,60 como indicadores de boa consistência interna, de acordo com Field. ${ }^{29}$

Finalmente, foi testada a adequação da solução factorial encontrada aos dados da amostra em estudo através de uma análise fatorial confirmatória com o método de Modelos de Equações Estruturais (software AMOS 19).

A comparação de estratégias e estilos de coping por género recorreu a testes $t$ para amostras independentes, com nível de significância de 0,05.

\section{RESULTADOS}

\section{Taxa de resposta e descrição da amostra}

Dos 2100 questionários entregues foram devolvidos 1 726. Destes, 13 foram excluídos por não corresponderem ao critério etário ou por apresentarem um padrão de respostas considerado susceptível de dúvida quanto à adequação/ veracidade das respostas, sendo a taxa de resposta final de $82 \%$.

A Tabela 1 caracteriza a amostra, comparando-a com os resultados sociodemográficos do maior estudo sobre comportamentos de saúde em adolescentes portugueses, o HBSC $2010,{ }^{30}$ sendo de salientar que este incluiu estudantes que frequentavam o sexto ano e não incluiu aqueles que frequentavam o $12^{\circ}$ ano. 
Tabela 1 - Dados sócio-demográficos da amostra, comparando com os dados nacionais do estudo HBSC 2010 (s.d. = sem dados comparáveis)

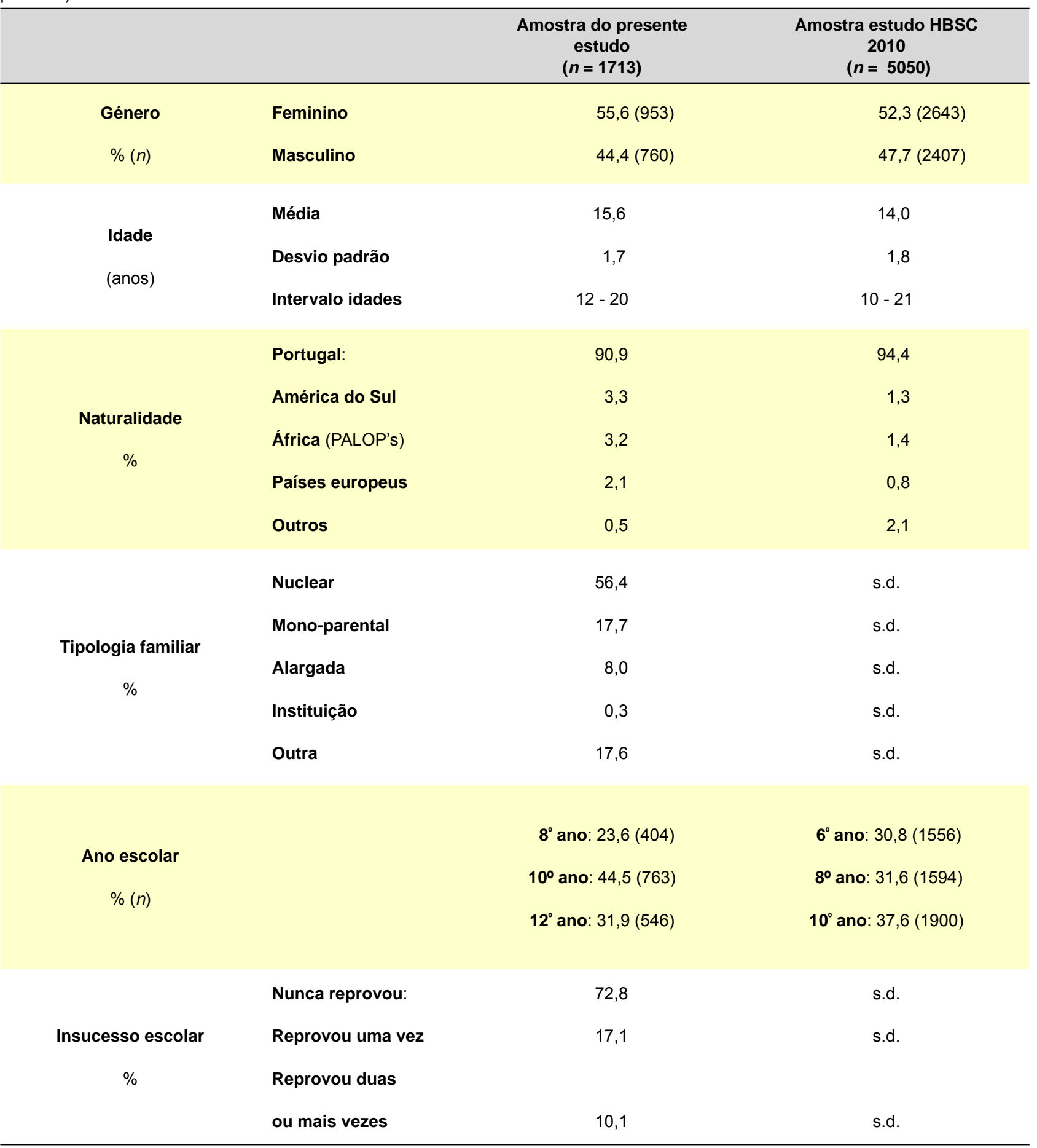

Análise em Componentes Principais (ACP-1, ACP-2 e ACP-3).

Tal como referido, a ACP foi realizada em três etapas, correspondendo a três grupos de itens selecionados aleatoriamente, tal como o procedimento adoptado pelos autores originais. Em todas as três etapas fizemos uma análise factorial exploratória com rotação direct oblimin e os itens que saturaram abaixo de 0,30 foram excluídos. Este tipo de rotação foi escolhido pois, segundo Field, ${ }^{29}$ é o tipo de rotação mais adequado a amostras de dimensão elevada e que permite uma distribuição mais livre dos itens em factores.

ACP 1 - A análise factorial exploratória sugeriu a retenção de 5 factores que explicam $49,32 \%$ da variância total (Tabela 2). A dimensão 'iludir-se' (adaptado do inglês 'wishful thinking') foi eliminada pois apenas dois itens saturaram nesse factor.

Verificámos que os factores de 'suporte social', 'culpabilizar-se', 'investir em amizades íntimas' mantiveram-se idênticos às mesmas dimensões da escala original. 
Tabela 2 A - Resultados das três etapas da Análise em Componentes Principais. ACP-1: KMO =0,85, Bartlett's test of sphericity X2 ${ }_{(351)}$ $=12088,88, p<0,001$

ACP -1

Dimensões

Itens

0,78

1

0,72

Suporte Social

Culpabilizar-se

42
51

35

47

62

Focar-se no positivo

69

65

13

40

77

Investir em amizades íntimas

53

11

14
2

Factores

3

4

5

0,86

0,85

0,74

0,58

0,75

0,73

0,52

$\underline{0,69}$

$\underline{0,50}$

$\underline{0,49}$

0,82

0,73

0,46

0,48

0,33
16

76

Ignorar o problema

58

0,77

0,62

$\underline{0,71}$

0,42

4,781

3,503

2,153

1,547

1,300

\% de variância

17,71

12,98

7,98

5,83

4,82

Cronbach $\alpha$

0,81

0,72

0,66

0,64 
Tabela 2 B - Resultados das três etapas da Análise em Componentes Principais. ACP-2: KMO = 0,79, Bartlett's test of sphericity $\mathrm{X}_{(351)}$ $=13352,129, p<0,001$

ACP-2

\begin{tabular}{|c|c|c|c|c|c|c|c|}
\hline \multirow[t]{2}{*}{ Dimensões } & \multirow[t]{2}{*}{ Itens } & \multicolumn{6}{|c|}{ Factores } \\
\hline & & 1 & 2 & 3 & 4 & 5 & 6 \\
\hline \multirow{5}{*}{ Preocupar-se } & 73 & 0,73 & & & & & \\
\hline & 7 & 0,70 & & & & & \\
\hline & 64 & 0,64 & & & & & \\
\hline & 24 & 0,60 & & & & & \\
\hline & 37 & 0,44 & & & & & \\
\hline \multirow{5}{*}{ Procurar apoio espiritual } & 48 & & 0,90 & & & & \\
\hline & 22 & & 0,89 & & & & \\
\hline & & & & & & & \\
\hline & 5 & & 0,85 & & & & \\
\hline & 36 & & 0,70 & & & & \\
\hline \multirow{3}{*}{ Guardar para si } & 57 & & & 0,85 & & & \\
\hline & 43 & & & 0,80 & & & \\
\hline & 15 & & & 0,63 & & & \\
\hline \multirow{4}{*}{$\begin{array}{l}\text { Procurar ajuda } \\
\text { profissional }\end{array}$} & 61 & & & & 0,86 & & \\
\hline & 72 & & & & 0,83 & & \\
\hline & 23 & & & & 0,80 & & \\
\hline & 6 & & & & 0,73 & & \\
\hline \multirow{5}{*}{$\begin{array}{l}\text { Concentrar-se na } \\
\text { resolução do problema }\end{array}$} & 2 & & & & & 0,83 & \\
\hline & 18 & & & & & 0,80 & \\
\hline & 78 & & & & & 0,67 & \\
\hline & 32 & & & & & 0,54 & \\
\hline & 44 & & & & & 0,52 & \\
\hline \multirow{4}{*}{ Reduzir a tensão } & 79 & & & & & & 0,51 \\
\hline & 27 & & & & & & 0,80 \\
\hline & 75 & & & & & & 0,54 \\
\hline & 68 & & & & & & 0,36 \\
\hline Eigenvalues & & 4,263 & 3,018 & 2,448 & 2,190 & 1,222 & 1,004 \\
\hline \% de variância & & 15,79 & 11,18 & 9,07 & 8,11 & 4,53 & 3,72 \\
\hline Cronbach $\alpha$ & & 0,72 & 0,86 & 0,69 & 0,82 & 0,73 & 0,55 \\
\hline
\end{tabular}


Tabela 2 C - Resultados das três etapas da Análise em Componentes Principais. ACP-3: KMO = 0,82, Bartlett's test of sphericity X2 ${ }_{(300)}$ $=8349,435, p<0,001$. Saturações em factores que não o previsto surgem sublinhadas

ACP-3

$\begin{array}{lll}\text { Dimensões } & \text { Itens } & \text { Factores }\end{array}$

5

$\begin{array}{ccc}33 & 0,73 \\ & 50 & 0,70 \\ \text { Procura de pertença } & 38 & 0,62 \\ & 8 & 0,55 \\ 46 & \underline{0,54}\end{array}$

52

0,72

67

0,68

Ação Social

49

0,67

39

0,57

66

$\underline{0,48}$

Não se confrontar

\section{Eigenvalues \\ \% de variância}

Cronbach $\alpha$

4,253
17,01
0,68

\section{1,679}

6,72

0,69

1,591

6,37

0,67
1,346

0,64
Na dimensão 'focar-se no positivo' registaram-se mais diferenças relativamente ao original. Dos seis itens que constituem esta dimensão, três pertenciam originalmente a outras dimensões (itens 13 e 69 provinham da dimensão 'iludir-se'; e o item 65 provinha de 'ignorar o problema').

Quanto à consistência interna das cinco dimensões enpondia a esta dimensão na escala original. 
contradas verificámos valores de razoáveis a bons.

ACP 2 - A análise factorial exploratória sugeriu a retenção de seis factores que explicam $52,4 \%$ da variância total (Tabela 2).

Todos os itens das dimensões 'preocupar-se', 'procurar apoio espiritual', 'guardar para si', 'procurar ajuda profissional', 'concentrar-se na resolução do problema' e 'reduzir a tensão' saturaram nos factores esperados, mantendo-se estas dimensões idênticas às descritas na estrutura original do instrumento. A única exceção remete para os factores 'guardar para si', em que o item 30 ('evito estar com pessoas') foi retirado por indicação estatística para aumentar a consistência interna da dimensão, tal como aconteceu relativamente à dimensão 'reduzir a tensão', em que o item 12 ('choro ou grito') foi também removido.

Quanto à consistência interna das seis dimensões encontradas verificamos valores Cronbach a aceitáveis, com a exceção de um valor baixo na dimensão 'reduzir a tensão'.

ACP 3 - A análise factorial exploratória sugeriu a retenção de cinco factores que explicam $46,7 \%$ da variância total
(Tabela 2).

Os resultados demonstram que apenas as dimensões de 'esforço e êxito' e 'atividade física' apresentam uma estrutura idêntica à encontrada na solução factorial original.

A dimensão 'atividades relaxantes' (adaptado do inglês 'seek relaxing diversions') foi eliminada pois apenas dois itens saturaram nesse factor. De notar que o item 46 (pertencente a esta dimensão na estrutura factorial original), se organizou no factor 1 - 'procura de pertença'. Quanto a esta última - 'procura de pertença' - todos os outros itens são idênticos aos identificados na estrutura original.

Quanto ao factor 'acção social', quatro dos seus cinco itens são idênticos à estrutura da dimensão original, excepto o item 66 ('faço o que os meus amigos querem') que originalmente corresponde à dimensão 'procura de pertença'.

A dimensão de 'não confronto', constitui-se apenas por três itens (no original esta dimensão constitui-se por cinco itens). No entanto, estes são idênticos aos que constituem a dimensão original. Os itens 63 ('sofro de dores de cabeça ou dores de estômago') e 28 ('fico doente ou agoniado') apresentaram uma saturação elevada num factor isolado

Tabela 3 - Resultados da Análise em Componentes Principais de todas as dimensões encontradas na adaptação portuguesa, com rotação varimax. Os 3 factores correspondem a 3 estilos de coping: factor 1 - focado na resolução do problema; factor 2 - referência a outros; factor 3 - coping não produtivo. KMO $=0,79$, Bartlett's test of sphericity $\chi^{2}{ }_{(120)}=7999,952, p<0,001$. Saturações em factores que não os previstos surgem sublinhadas.

\begin{tabular}{|c|c|c|c|}
\hline \multirow[t]{2}{*}{ Dimensões } & \multicolumn{3}{|c|}{ Factores } \\
\hline & 1 & 2 & 3 \\
\hline Concentrar-se na resolução do problema & 0,79 & & \\
\hline Esforçar-se e ter êxito & 0,75 & & \\
\hline Preocupar-se & $\underline{0,75}$ & & \\
\hline Procura de pertença & 0,60 & & \\
\hline Focar-se no positivo & 0,56 & & \\
\hline Fazer atividade física & 0,35 & & \\
\hline Ação Social & & 0,76 & \\
\hline Investir em amizades íntimas & & $\underline{0,63}$ & \\
\hline Procurar ajuda profissional & & 0,62 & \\
\hline Suporte Social & & 0,61 & \\
\hline Procurar apoio espiritual & & 0,31 & \\
\hline Ignorar o problema & & & 0,77 \\
\hline Guardar para si & & & 0,74 \\
\hline Culpabilizar-se & & & 0,72 \\
\hline Não se confrontar & & & 0,69 \\
\hline Reduzir a tensão & & & 0,51 \\
\hline Eigenvalues & 4,011 & 2,667 & 1,443 \\
\hline \% de variância & 18,81 & 16,38 & 15,63 \\
\hline Cronbach $\alpha$ & 0,76 & 0,61 & 0,74 \\
\hline
\end{tabular}




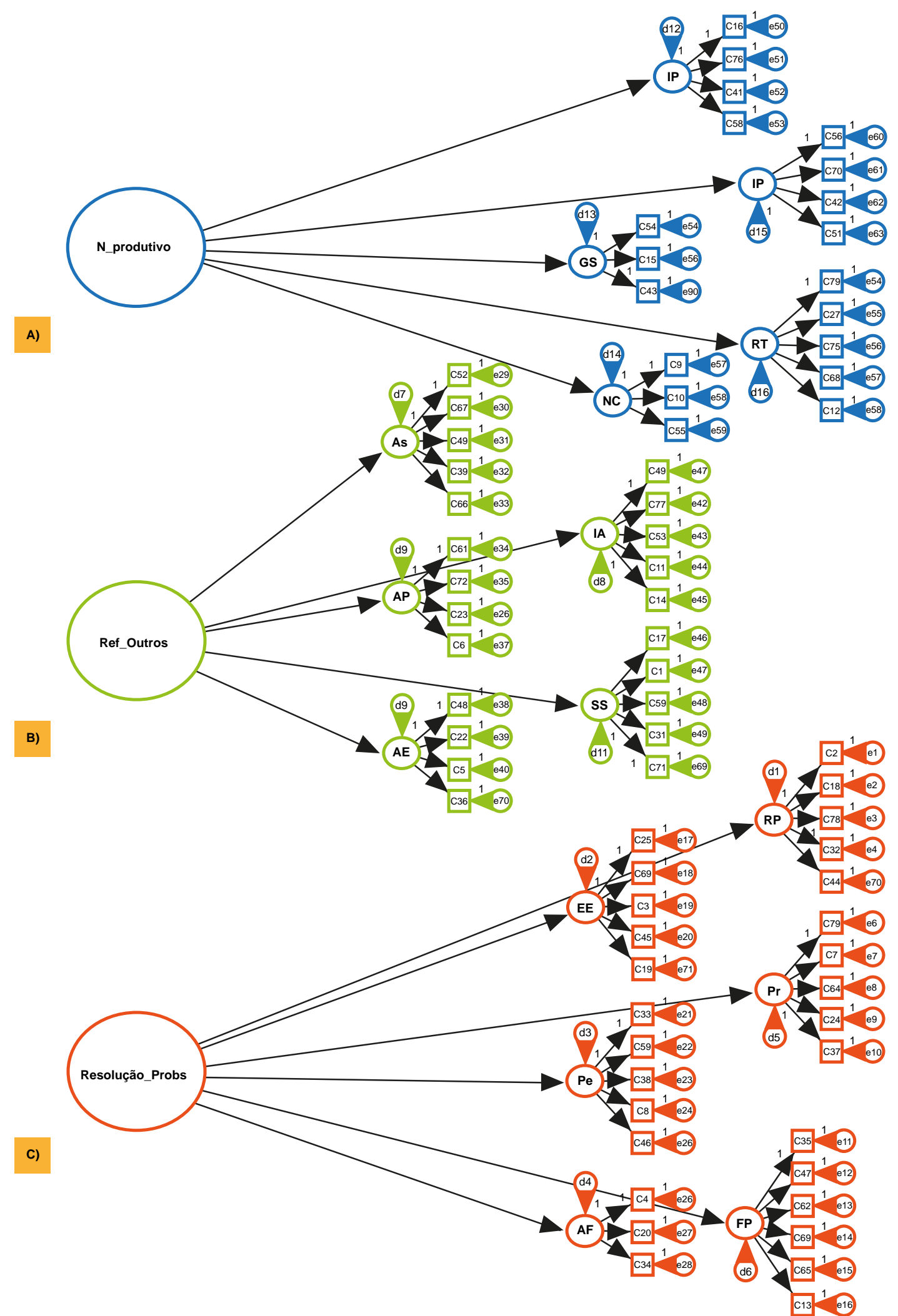

Figura 1 - Modelo estrutural proposto para a ACS- versão Portuguesa. Observam-se os resultados obtidos para cada uma das dimensões de estilos de coping. Todos os índices de ajustamento apresentam valores de $p<0,001$.

A) estrutura factorial proposta para a dimensão de coping 'não produtivo'- $\mathrm{X} 2(129)=809,53, p<0,001, \mathrm{CFI}=0,92, \mathrm{GFI}=0,95, \mathrm{NFI}=$ 0,90 , e RMSEA = 0,06.

B) estrutura factorial proposta para a dimensão de coping 'referência a outros' $\mathrm{X} 2(210)=846,23, p<0,001, \mathrm{CFI}=0,95, \mathrm{GFI}=0,96, \mathrm{NFI}$ $=0,94$, e RMSEA = 0,04.

C) estrutura factorial proposta para a dimensão de coping 'resolução de problemas' - $\mathrm{X} 2(335)=1421,65, p<0,001, \mathrm{CFI}=0,92, \mathrm{GFI}=$ $0,94, \mathrm{NFI}=0,90$, e RMSEA $=0,04$. 
apelando para uma dimensão mais associada a somatização. No entanto, por se tratar de um factor muito específico e com apenas dois itens, este foi excluído da solução factorial, por motivos de parcimónia, dada a dimensão já elevada da escala em estudo.

Quanto à consistência interna das cinco dimensões encontradas verificamos valores Cronbach a aceitáveis, demonstrando que as dimensões encontradas apresentam boa consistência.

\section{Análise factorial de segunda ordem}

Seguidamente, foi realizada uma segunda ACP, com rotação varimax, que sugeriu a organização dos factores de primeira ordem em três factores mais abrangentes (critério de Kaiser superior a 1) que explicam $50,8 \%$ da variância total (Tabela 3). Tratando-se de uma análise de segunda ordem, a semelhança entre os factores desta solução factorial partilhariam certamente mais comunalidades, pelo que se optou por uma rotação ortogonal, varimax, que maximiza a dispersão das saturações dos itens ${ }^{29}$ e, como tal, enfatiza as diferenças entre os factores encontrados. Este tipo de rotação foi também utilizado pelos autores originais da escala.

As 16 estratégias de coping da versão adaptada agruparam-se em três estilos de coping, de acordo com a versão original da $\mathrm{ACS},{ }^{20}$ focado na resolução do problema; referência a outros, e coping não produtivo.

Todas se agruparam nos factores previstos de acordo com a estrutura original, excepto duas: 'preocupar-se', que na versão original da escala pertencia ao estilo de coping não produtivo e; 'investir em amizades íntimas' que originalmente estava no estilo de coping focado na resolução do problema. Quanto à consistência interna das três dimensões encontradas verificámos valores Cronbach $\alpha$ aceitáveis, reveladores de elevada consistência interna destes agrupamentos.

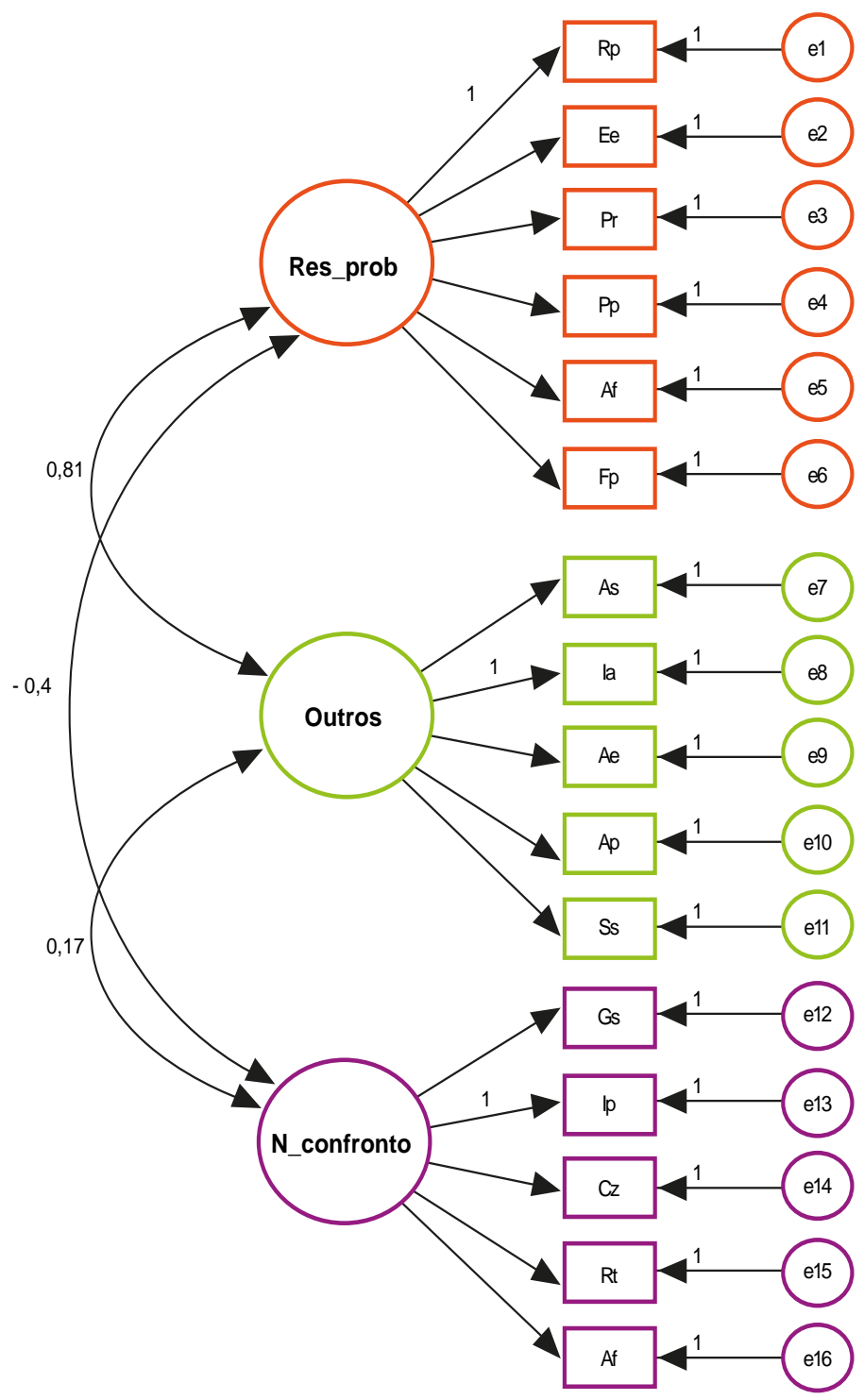

Figura 2 - Modelo estrutural proposto para a ACS - versão Portuguesa. Observam-se os resultados obtidos para estrutura global da escala. Todos os índices de ajustamento apresentam valores de $p<0,001$. Estrutura global $-\mathrm{X} 2(62)=579,23, p<0,001, \mathrm{CFI}=0,94, \mathrm{GFI}=$ $0,96, \mathrm{NFI}=0,93$, e RMSEA $=0,07$. 


\section{Análise factorial confirmatória}

Após identificarmos a estrutura factorial da versão Portuguesa da ACS (16 dimensões, correspondentes a estratégias de coping e organizadas em três factores mais abstractos, correspondentes a estilos de coping), conduzimos uma análise factorial confirmatória para verificar a adequação (fit) da mesma aos dados em estudo. A análise foi realizada com recurso ao método de Modelos Equações Estruturais, com método maximum likelihood, para a análise da matriz de covariâncias e das correlações entre dimensões. O ajustamento do modelo foi examinado com recurso a índices de ajustamento reconhecidos como bons indicadores de fit com amostras grandes de indivíduos, nomeadamente: CFI (comparative fit index), GFI (godness-of-fit index), $\mathrm{NFI}$ (normed fit index), cujos valores acima de 0,90 indicam um óptimo ajustamento do modelo; e o RMSEA (root mean square error of aproximation), cujos valores indicadores de bom ajustamento devem ser iguais ou inferiores a 0,10.
As estruturas dos três estilos de coping foram analisadas separadamente e, num segundo passo foi analisada a estrutura global. Os resultados demonstraram que, quer cada uma das dimensões de estilos de coping, quer a estrutura global apresentam bons índices de ajustamento aos dados (Fig.s 1 e 2).

\section{Estratégias e estilos de coping por sexo}

$\mathrm{Na}$ nossa amostra verificámos que as estratégias mais utilizadas pelos adolescentes são: 'preocupar-se'; 'concentrar-se na resolução do problema' e; 'esforçar-se e ter êxito'. Segundo o nosso modelo todas estas se englobam no estilo de coping focado na resolução do problema. Como podemos observar na Tabela 4 existem diferenças de sexo quanto às estratégias de coping utilizadas.

Os adolescentes do sexo masculino recorrem comparativamente mais às seguintes estratégias: 'ação social'; 'fazer atividade física' e; 'ignorar o problema'. Por outro

Tabela 4 - Comparações da utilização de estratégias e estilos de coping por sexo, através do teste T de Student para amostras independentes. A negrito encontram-se aquelas em que encontrámos diferenças significativas. (DP = desvio padrão; n.s. = não significativo).

\begin{tabular}{|c|c|c|c|c|c|}
\hline \multirow[b]{2}{*}{ Estratégia } & \multicolumn{2}{|c|}{$\begin{array}{l}\text { Masculino } \\
(n=586)\end{array}$} & \multicolumn{2}{|c|}{$\begin{array}{c}\text { Feminino } \\
(n=755)\end{array}$} & \multirow[b]{2}{*}{$p$} \\
\hline & Média & DP & Média & DP & \\
\hline Ação Social & 1,62 & 0,59 & 1,56 & 0,54 & 0,014 \\
\hline Concentrar-se na resolução do problema & 3,63 & 0,72 & 3,67 & 0,67 & 0,21 \\
\hline Culpabilizar-se & 2,49 & 0,92 & 2,74 & 0,98 & $<0,001$ \\
\hline Esforçar-se e ter êxito & 3,58 & 0,65 & 3,61 & 0,64 & 0,20 \\
\hline Fazer atividade física & 3,53 & 0,96 & 3,11 & 0,88 & $<0,001$ \\
\hline Focar-se no positivo & 3,20 & 0,73 & 3,14 & 0,74 & 0,13 \\
\hline Guardar para si & 2,80 & 0,97 & 2,72 & 0,97 & 0,11 \\
\hline Ignorar o problema & 2,11 & 0,77 & 2,02 & 0,74 & 0,01 \\
\hline Investir em amizades íntimas & 2,93 & 0,89 & 2,91 & 0,84 & 0,60 \\
\hline Não se confrontar & 1,94 & 0,78 & 1,92 & 0.79 & 0,72 \\
\hline Preocupar-se & 3,77 & 0,75 & 3,96 & 0,69 & $<0,001$ \\
\hline Procura de pertença & 3,49 & 0,75 & 3,62 & 0,70 & $<0,001$ \\
\hline Procurar ajuda profissional & 1,78 & 0,82 & 1,74 & 0,84 & 0,44 \\
\hline Procurar apoio espiritual & 1,84 & 1,01 & 2,00 & 1,04 & 0,001 \\
\hline Reduzir a tensão & 1,86 & 0,64 & 2,25 & 0,70 & $<0,001$ \\
\hline Suporte Social & 2,80 & 0,82 & 3,16 & 0,85 & $<0,001$ \\
\hline Estilo geral & Média & DP & Média & DP & $p$ \\
\hline Focado na resolução do problema & 3,54 & 0,52 & 3,53 & 0,49 & 0,77 \\
\hline Referência a outros & 2,18 & 0,53 & 2,26 & 0,52 & 0,002 \\
\hline Não produtivo & 2,24 & 0,58 & 2,32 & 0,59 & 0,006 \\
\hline
\end{tabular}


lado, as adolescentes do sexo feminino utilizam com maior frequência as seguintes: 'culpabilizar-se'; 'preocupar-se'; 'procura de pertença'; 'procurar apoio espiritual'; "'eduzir a tensão'; e 'suporte social'.

Quanto aos estilos de coping verificámos que o focado na resolução do problema é aquele maioritariamente utilizado pelos adolescentes na nossa amostra. As diferenças relativas ao sexo foram significativas para os estilos de coping não produtivo e referência a outros, ambos com valores médios mais elevados no sexo feminino.

\section{DISCUSSÃO}

A taxa de resposta deste estudo foi surpreendentemente positiva, o que possibilitou a realização da metodologia estatística proposta. A nossa abordagem permitiu manter uma estrutura muito semelhante à original: um total de 70 itens que avaliam 16 estratégias de coping e agrupando-se em três estilos gerais. No sentido de reforçar a validade dos resultados obtidos servimo-nos dos resultados sociodemográficos do maior estudo sobre comportamentos de saúde em adolescentes portugueses, o HBSC 201030 (também realizado em adolescentes que frequentavam estabelecimentos de ensino público), para verificar as semelhanças com a nossa amostra. A partir desta observação (Tabela 1) considerou-se existirem francas semelhanças a nível sociodemográfico entre amostras que indiciam uma boa adequação da amostra utilizada.

Os resultados da análise em componentes principais de primeira ordem (realizada em três etapas), sugeriu uma estrutura de 16 dimensões (estratégias de coping), menor que a estrutura original constituída por 18 dimensões. Estas diferenças poderão ser atribuídas às significações diferenciais impostas pela heterogeneidade cultural existente entre os jovens da amostra do estudo original e a amostra Portuguesa. Como referido na introdução, estudos prévios indicam que diferenças culturais, geográfica e socioeconó-

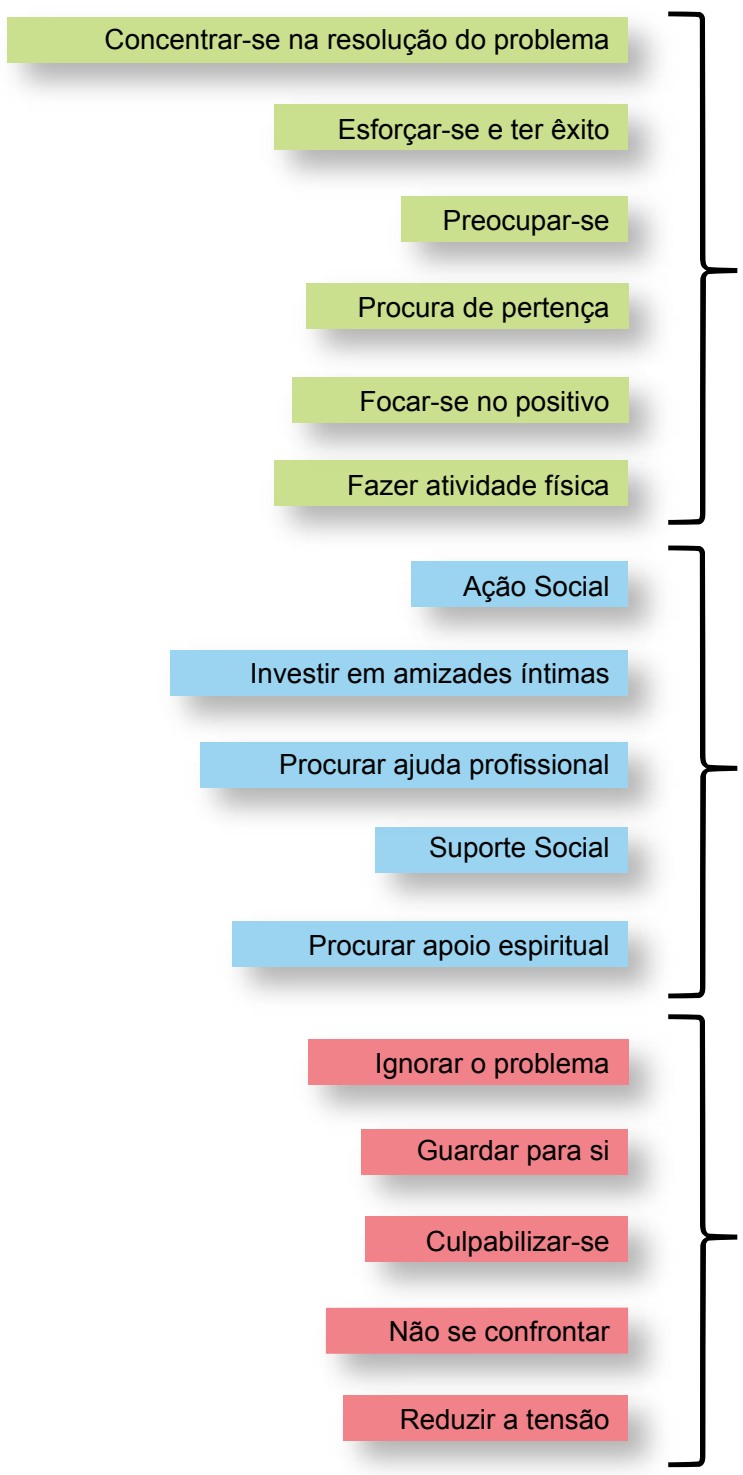

Focado na resolução do problema (mantendo-se socialmente conectado e em forma física)

Referência a outros

(ou pedido de ajuda a outros para lidar com o problema)

Figura 3 - Esquematização da versão adaptada para português da ACS. Na coluna da esquerda estão nomeadas as 16 estratégias de coping e a forma como se agrupam nos 3 estilos de coping (na coluna da direita). 
micas influem nas variáveis de coping..$^{10-12}$

No nosso estudo não foram identificadas as dimensões 'iludir-se' (adaptado do inglês 'wishful thinking') e 'procurar atividades relaxantes' (adaptado do inglês 'seek relaxing diversions'). Também no estudo de validação da ACS versão em Espanhol da Argentina ${ }^{22}$ a dimensão 'wishful thinking' foi eliminada. A literatura não oferece explicações claras para este fenómeno e por isso hipotetizámos que este conceito não tem uma significação clara no contexto Português e, por isso, os itens desta dimensão adquirem significações mais coerentes com outras dimensões de coping (ex. 'pensar de forma positiva', 'ignorar o problema') nos jovens Portugueses em estudo.

Por outro lado, os jovens Portugueses da amostra em estudo parecem considerar as atividades de lazer como de carácter fortemente social, dado que esta amostra da nossa população parece organizar conjuntamente conteúdos de ativação da rede social e conteúdos relativos a atividades relaxantes, não distinguindo estas últimas das anteriores. As atividades de lazer parecem então reforçar e contribuir para a procura/ aproximação dos outros e, consequentemente, o fortalecimento da rede social, aspecto fulcral para um coping adequado. ${ }^{31}$

Importa referir um resultado interessante, que ocorreu também no estudo de replicação da ACS original ${ }^{16} \mathrm{em}$ que os autores observaram que a escala 'não se confrontar' se separou em dois factores: 'somatização' e 'desespero'. Também no nosso estudo isso aconteceu e levou à eliminação de dois itens que se agruparam individualmente e que descreviam sintomas de somatização.

Quanto à análise factorial de segunda ordem foram encontrados os estilos de coping esperados e globalmente as estratégias agruparam-se de acordo com o modelo original, com a exceção das estratégias 'preocupar-se', que na versão original da escala estava no estilo de coping não produtivo, e 'investir em amizades íntimas' que originalmente estava no estilo de coping focado na resolução do problema. Estas duas diferenças apresentam bastante suporte estatístico (com altos valores de saturação) embora de um ponto de vista teórico a sua justificação seja desafiante.

Quanto à estratégia 'preocupar-se', esta saturou de forma inequívoca no factor de coping produtivo (factor $1=$ 0,753 ; factor $2=0,158$ e factor $3=0,111$ ). Se bem que estatisticamente não exista lugar a dúvidas, do ponto de vista teórico a discussão torna-se mais complexa pois sendo, de facto, um primeiro passo para resolver os problemas, ${ }^{32}$ na maior parte dos estudos verifica-se que com o aumento dos valores de preocupação existe um proporcional défice na 'orientação do problema', a parte inicial do processo comportamental de resolução de problemas ${ }^{32,33}$ (no fundo a presença de preocupação excessiva ou patológica, pode levar a que a pessoa não se motive para a sua resolução). É claro que existem graus distintos de preocupação e muitos dos adolescentes, ao responder a este item, poderão não estar a pensar em preocupação 'patológica' ou 'não produtiva'. É também muito relevante discutir que existem certas capacidades cognitivas que são necessárias para o processo cognitivo de preocupação e que só poderão estar desenvolvidas no final da adolescência, ${ }^{34}$ pelo que a distinção entre preocupação como primeiro passo de resolução do problema ou preocupação excessiva poderá não ser clara em muitos dos jovens da nossa amostra, o que poderá ter levado ao resultado obtido.

Quanto à estratégia 'investir em amizades íntima'”, o seu reposicionamento apresenta forte base estatística, com uma saturação bastante mais acentuada no factor de referência a outros (factor $2=0,631$ ), embora com um crossloading moderado com o factor de coping focado na resolução do problema (factor $1=0,322$ ). Do ponto de vista teórico pareceu-nos perfeitamente aceitável a sua inclusão no estilo de coping de referência a outros.

Os três estilos de coping encontrados no nosso estudo são conceptualmente semelhantes, com a exceção da dimensão preocupar-se, aos descritos originalmente na ACS e mencionados na introdução, o que fortalece os resultados encontrados, sugerindo a adequação da solução factorial encontrada para a população Portuguesa. A Fig. 3 resume a estrutura da versão adaptada para Português da ACS.

A adequação desta estrutura é corroborada pelos restantes resultados preliminares sugeridos pelo presente estudo, nomeadamente:

a) a análise factorial confirmatória que parece revelar um bom fit da estrutura da medida aos dados da amostra em estudo;

b) os valores de consistência interna (alfa de Cronbach) das dimensões de primeira e de segunda ordem;

c) as diferenças entre sexos identificadas, semelhantes a estudos prévios.

Quando à consistência interna, todos os valores encontrados estão acima de 0,60 (variando entre 0,63 e 0,86), excepto na dimensão 'redução de tensão', que apresentou um valor de 0,55. Mantivemos contudo esta dimensão pois, em estudos exploratórios de medidas psicológicas complexas (como é o caso do coping), e em escalas com grande número de itens valores de consistência interna acima de 0,50 são habitualmente considerados. ${ }^{4}$ Concretamente, neste tipo de estudos os valores de consistência interna não ultrapassam, regra geral, os valores obtidos na presente adaptação. No estudo de replicação da ACS, ${ }^{16}$ os autores optaram por manter a dimensão 'procurar atividades relaxantes' apesar de um alfa de Cronbach de 0,59, sendo importante realçar que se trata da população original (adolescentes Australianos) de onde origina esta medida. Outros estudos de adaptação da ACS revelam valores de consistência interna um pouco inferiores a 0,6022.

Para além disso, esta parece ser uma limitação transversal a várias escalas de medição de coping. No estudo de adaptação para Português da escala Brief COPE, ${ }^{35}$ uma dimensão apresentou um valor de 0,55. Também na adaptação Portuguesa da escala Ways of Coping Questionnai$r e,{ }^{35}$ verificaram-se duas dimensões com Cronbach $\alpha$ ligeiramente abaixo de 0,60 .

Importa ainda referir que os valores de consistência interna são mais elevados em medidas baseadas em even- 
tos específicos, ${ }^{36}$ contrariamente ao que sucede nesta escala cuja instrução remete para as estratégias de coping em geral, e não são centradas numa determinada situação.

Quanto ao efeito do sexo, verificámos que os rapazes e as raparigas tendem para diferentes estratégias de coping, parecendo as jovens mais focadas em estratégias de ativação da rede social do que os rapazes, mas também em estratégias mais emocionais como culpabilizar-se ou reduzir a tensão. Por outro lado, os adolescentes do sexo masculino utilizam comparativamente mais estratégias ativas - recurso a ação social, atividade física - ou então optam por ignorar o problema. Estes dados estão de acordo com o que é descrito na literatura, ${ }^{8,9}$ sendo que estas similaridades reforçam a nosso ver a hipótese de adequação da estrutura final encontrada no presente estudo.

Realçamos de seguida as principais limitações deste estudo.

Sendo o coping um conceito complexo, são esperadas variações conforme as populações estudadas, devido a influências sociais, culturais ou mesmo variações de origem mais estrutural/ biológica, ${ }^{4}$ neste contexto é importante referir que os dados neste estudo refletem apenas a realidade dos jovens que frequentam o ensino público numa zona metropolitana de uma grande cidade portuguesa (Lisboa). Assim sendo é possível a existência de diferenças relativas às populações de outras áreas urbanas, mas é sobretudo expectável, de acordo com a literatura, ${ }^{11}$ que se observem diferenças em amostras de adolescentes provenientes de meio rural. Outra limitação prende-se com a exclusão de estabelecimentos de ensino privado, que poderia influenciar os resultados noutra direção, como foi por exemplo verificado num estudo de uma grande amostra de adolescentes (utilizando a versão Espanhola da ACS) ${ }^{12}$ em que os jovens do ensino público e privado apresentaram resultados diferentes.

Os dados apresentados representam uma parte do protocolo de validação Portuguesa da escala, nomeadamente a sua adaptação linguística e estudo da consistência interna e da estrutura fatorial. Como referido anteriormente, a aplicação da ACS foi incluída num questionário criado no âmbito de um estudo mais abrangente ${ }^{28} \mathrm{e}$, por razões logísticas, foi necessário planear para uma segunda fase o restante estudo das validades de constructo e de critério (protocolo que irá incluir um conjunto de instrumentos adequados ao estudo da validade convergente-discriminante). Por esta razão, a utilização desta escala adaptada deve ser cuidadosamente ponderada e os seus resultados interpretados com prudência. Isto torna-se particularmente relevante no caso das dimensões que revelaram valores mais baixos de consistência interna.

Futuramente, iremos aprofundar o estudo da sua validade convergente-discriminante do instrumento bem como

\section{REFERÊNCIAS}

1. Lazarus R, Folkman S. Stress, appraisal, and coping. New York: Springer Publishing Company; 1984.

2. Holahan CJ, Moos RH. Personal and contextual determinants of coping strategies. J Pers Soc Psychol. 1987;52:946-55. o efeito da idade, no sentido de melhor compreender a capacidade discriminativa de escala e o seu comportamento psicométrico.

\section{CONCLUSÃO}

O presente estudo resultou na criação de uma versão adaptada para Português de um importante instrumento de avaliação de coping em adolescentes, a ACS, sendo que os resultados obtidos demonstraram valores psicométricos muito satisfatórios.

A nossa abordagem permitiu manter uma estrutura muito semelhante à original: um total de 70 itens que avaliam 16 estratégias de coping e se agrupam em três estilos de coping, estrutura para a qual consideramos existirem fundamentos plausíveis quanto à sua adequabilidade na avaliação do coping em adolescentes Portugueses.

Aparte das limitações inerentes a este estudo consideramos que o presente trabalho representa uma contribuição válida e importante para o estudo do coping com adolescentes Portugueses.

\section{AGRADECIMENTOS}

Os autores agradecem à Fundação para a Ciência e Tecnologia (FCT) por financiar parcialmente este estudo. Agradecem também ao Ministério da Educação pela colaboração prestada e, em especial, a Izabel Baptista. Expressamos também o nosso profundo agradecimento às escolas e professores que connosco colaboraram e, claro, aos estudantes e aos seus encarregados de educação sem os quais este estudo não teria sido possível ou relevante. Agradecemos também a Inês Mirra pelo apoio no trabalho de campo e Anne Rodrigues pelo apoio na questão linguística. O último agradecimento é dirigido a Erica Frydenberg que, à distância, nos apoiou na adaptação linguística mas também na discussão teórica.

\section{CONFLITOS DE INTERESSE}

Os autores declaram não ter quaisquer conflitos de interesse relativamente ao presente artigo. Por razões de copyright não nos é possível apresentar em anexo a versão adaptada para português da $\mathrm{ACP}$, que será enviada para a editora proprietária da ACS (Australian Council for Educational Research), que a disponibilizará para os investigadores interessados.

\section{FONTES DE FINANCIAMENTO}

Diogo Frasquilho Guerreiro recebeu uma bolsa de investigação da FCT que foi utilizada como fonte de financiamento parcial para este estudo. (FRH/SINTD/60041/2009). Para além desta, não existiram outras fontes externas de financiamento.

3. Lazarus RS. Coping theory and research: past, present, and future. Psychosom Med. 1993;55:234-47

4. Frydenberg E. Adolescent coping: advances in theory, research and practice. London: Routledge; 2008. 
5. Seiffge-Krenke I. Stress, coping and relationships in adolescence. New Jersey: Lawrence Erlbaum Associates; 1995.

6. Sampaio D. Lavrar o mar. Lisboa: Editorial Caminho; 2006.

7. Shaffer D, Kipp K. Developmental psychology - childhood \& adolescence. Belmont: Wadworth; 2007.

8. Frydenberg E, Lewis R. Boys play sport and girls turn to others: age, gender and ethnicity as determinants of coping. J Adolesc. 1993;16:25366.

9. Borges AI, Manso DS, Tomé G, Matos MG. Ansiedade e coping em crianças e adolescentes: Diferenças relacionadas com a idade e género. Análise Psicol. 2008;4:551-61.

10. Frydenberg E, Lewis R, Kennedy G, Ardila R, Wolfgang F, Rasmiyah $\mathrm{H}$. Coping with concerns: an exploratory comparison of Australian, Colombian, German, and Palestinian adolescents. J Youth Adolescence. 2003;32:59-66.

11. Elgar FJ, Arlett C, Groves R. Stress, coping, and behavioural problems among rural and urban adolescents. J Adolesc. 2003;26:577-88.

12. de la Paz Bermudez M, Teva I, Buela-Casal G. Influencia de variables sociodemograficas sobre los estilos de afrontamiento, el estres social y la busqueda de sensaciones sexuales en adolescentes. Psicothema. 2009;21:220-6

13. Davis SK, Humphrey N. The influence of emotional intelligence (EI) on coping and mental health in adolescence: divergent roles for trait and ability El. J Adolesc. 2012;35:1369-79.

14. Rao K. Recent research in stress, coping and women's health. Curr Opin Psychiatry. 2009;22:188-93.

15. Compas BE, Jaser SS, Dunn MJ, Rodriguez EM. Coping with chronic illness in childhood and adolescence. Annu Rev Clin Psychol. 2012;8:455-80.

16. Frydenberg $\mathrm{E}$, Lewis $\mathrm{R}$. A replication study of the structure of the adolescent coping scale: multiple forms and applications of a self-report inventory in a counselling and research context. Eur J Psychol Assessment. 1996;12:224-35.

17. Folkman S, Moskowitz JT. Coping: pitfalls and promise. Annu Rev Psychol. 2004;55:745-74

18. Compas BE, Connor-Smith JK, Saltzman H, Thomsen AH, Wadsworth ME. Coping with stress during childhood and adolescence: problems, progress, and potential in theory and research. Psychol Bull. 2001;127:87-127.

19. Moos RH, Billings AG. Conceptualising and measuring coping resources and processes. In: Goldberger L, Brenitz S, editors. Handbook of stress: Theoretical and clinical aspects. New York: Free Press; 1982. p. 212-30.

20. Frydenberg E, Lewis R. Adolescent Coping Scale: administrator's manual. Melbourne: Austr Council Educl Res; 1993.
21. Frydenberg E, Lewis R. Escala de afrontamiento para adolescentes. Madrid: TEA; 1996.

22. Richaud de Minzi MC. Coping assessment in adolescents. Adoles cence. 2003;38:321-30.

23. Leclercl D, Pronovost J, Dumont M. Échelle de Coping pour Adolescent: validation cannadienne-française de l'Adolescent Coping Scale de Frydenberg et Lewis (1993). Rev Quebecoise Psychol. 2009;30:177-96.

24. Câmara SG, Castellá SJ, Remor EA. Análise fatorial da escala de afrontamento para adolescentes (ACS) em uma amostra de jovens de Porto Alegre. Aletheia. 2002:15-31.

25. Omar K, Bujang MA, Mohd DT, et al. Validation of the Malay version of Adolescent Coping Scale. Int Medical J. 2011;18:288-92.

26. Hawton K, Rodham K, Evans E, Weatherall R. Deliberate self harm in adolescents: self report survey in schools in England. BMJ. 2002;325:1207-11.

27. O'Connor RC, Rasmussen S, Miles J, Hawton K. Self-harm in adolescents: self-report survey in schools in Scotland. $\mathrm{Br} \mathrm{J}$ Psychiatry. 2009;194:68-72.

28. Guerreiro DF. Comportamentos auto-lesivos em adolescentes em idade escolar: a sua relação com mecanismos de coping, temperamento e psicopatologia. Lisboa: Faculdade de Medicina da Universidade de Lisboa; 2014.

29. Field AP. Discovering statistics using SPSS. 3rd ed. Los Angeles: SAGE Publications; 2009.

30. Matos MG, Simões C, Tomé G, Camacho I, Ferreira M, Ramiro L. A saúde dos adolescentes portugueses: Relatório do estudo HBSC 2010. Lisboa: Centro de Malária e Outras Doenças Tropicais /IHMT/UNL 2012.

31. Trainor S, Delfabbro P, Anderson S, Winefield A. Leisure activities and adolescent psychological well-being. J Adolesc. 2010;33:173-86.

32. Dugas M, Letarte H, Rhéaume J, Freeston M, Ladouceur R. Worry and problem solving: Evidence of a specific relationship. Cognitive Therapy Res. 1995;19:109-20.

33. Laugesen N, Dugas MJ, Bukowski WM. Understanding adolescent worry: the application of a cognitive model. J Abnorm Child Psychol. 2003;31:55-64.

34. Vasey M. Development and cognition in childhood anxiety: The example of worry. In: Ollendick T, Prinz R, editors. Advances in clinical child psychology. New York: Plenum; 1993, p.1-39.

35. Pais-Ribeiro J, Rodrigues AP. Questões acerca do coping: a propósito do estudo de adaptação do Brief Cope. Psicol Saúde Doenças. 2004;5:3-15.

36. Carver CS, Scheier MF, Weintraub JK. Assessing coping strategies: a theoretically based approach. J Pers Soc Psychol. 1989;56:267-83. 


\section{Estudo de Adaptação e Características Psicométricas da Versão Portuguesa da Adolescent Coping Scale - Escala de \\ Coping para Adolescentes}

Publicado pela Acta Médica Portuguesa, a Revista Científica da Ordem dos Médicos

Av. Almirante Gago Coutinho, 151

1749-084 Lisboa, Portugal.

Tel: +351218428215

E-mail: submissao@actamedicaportuguesa.com

www.actamedicaportuguesa.com

ISSN:0870-399X | e-ISSN: 1646-0758

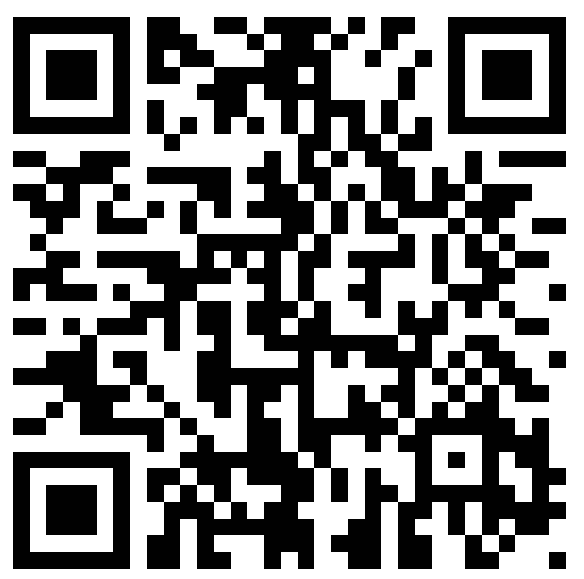

